\title{
Real Time Intraoperative Monitoring of Blood Loss with a Novel Tablet Application
}

\author{
Behnam Sharareh ${ }^{1}$, Spencer Woolwine ${ }^{1}$, Siddarth Satish ${ }^{2}$, Peter Abraham ${ }^{1}$ and Ran Schwarzkopf ${ }^{*}, 1$ \\ ${ }^{I}$ Department of Orthopaedic Surgery, Joint Replacement Service, University of California, Irvine, Orange, CA, USA \\ ${ }^{2}$ Gauss Surgical, Inc. Los Altos, CA, USA
}

\begin{abstract}
Introduction: Real-time monitoring of blood loss is critical in fluid management. Visual estimation remains the standard of care in estimating blood loss, yet is demonstrably inaccurate. Photometric analysis, which is the referenced "gold-standard" for measuring blood loss, is both time-consuming and costly. The purpose of this study was to evaluate the efficacy of a novel tablet-monitoring device for measurement of $\mathrm{Hb}$ loss during orthopaedic procedures.

Methods: This is a prospective study of 50 patients in a consecutive series of joint arthroplasty cases. The novel System with Feature Extraction Technology was used to measure the amount of $\mathrm{Hb}$ contained within surgical sponges intraoperatively. The system's measures were then compared with those obtained via gravimetric method and photometric analysis. Accuracy was evaluated using linear regression and Bland-Altman analysis.

Results: Our results showed a significant positive correlation between Triton tablet system and photometric analysis with respect to intra-operative hemoglobin and blood loss at 0.92 and 0.91 , respectively.

Discussion: This novel system can accurately determine $\mathrm{Hb}$ loss contained within surgical sponges. We believe that this user-friendly software can be used for measurement of total intraoperative blood loss and thus aid in a more accurate fluid management protocols during orthopaedic surgical procedures.
\end{abstract}

Keywords: Fluid management, gravimetric method, hemoglobin loss, intraoperative blood loss, photometric analysis, total joint arthroplasty.

\section{INTRODUCTION}

Currently there is a lack of a standardized approach of objectively measuring intraoperative blood loss that is accurate and can be completed in real time. Blood is gathered intraoperatively with the use of surgical sponges and vacuum suction, however, confounding variables like saline, bodily fluids or tissues, and intravascular volume status (hemodilution, hemoconcentration, and third body spacing) are rarely accounted for in calculating intraoperative blood loss. We are left with three commonly cited strategies for quantifying surgical blood loss: (1) Subjective assessment by physicians, (2) gravimetric analysis, and (3) photometric analysis. Subjective measurement by physicians is the most commonly used method due to convenience and efficiency. It relies on the surgeon's or anesthesiologist's calculation of hemorrhage and other fluid loss, blood captured via surgical sponges, vacuum suction canister, blood on the floor and other surfaces, and amount of fluid is used for irrigation intraoperatively. It becomes clear that taking these numerous variables into consideration can be extremely challenging and likely the reason why subjective assessment is inaccurate and unreliable [1-7]. It has also shown that surgical

*Address correspondence to this author at the Joint Replacement Service, Orthopaedic Department, University of California, Irvine, 101 The City Drive South, Orange, CA 92868, USA; Tel: 714-456-5759;

E-mail: schwarzk@gmail.com experience of the individual has had little impact on improving accuracy in estimating blood loss [8].

Gravimetric analysis is a relatively simple concept that assumes $1 \mathrm{~mL}$ of blood equals 1 gram, and the dry weights of surgical sponges are subtracted from the wet weights of blood soaked sponges. This method is also not immune to confounding variables like saline irrigation, suctioned devices, other body fluids, and solid body tissues that can get captured by surgical sponges and weighed at the end, resulting in yet another unreliable method $[5,9,10]$. Photometric analysis and laboratory testing have been shown to be reliable techniques for measuring blood loss in the research setting and are currently discussed as the "gold standard" for accurately quantifying blood loss $[11,12]$. The process involves rinsing and assaying blood soaked products to determine hemoglobin concentration through spectrophotometry and intraoperative laboratory work-up of a patient's blood samples. These processes are laborious, time consuming and costly ways to monitor blood loss and as a result are rarely performed. Therefore, a dependable, real-time technique for direct measurement of estimated surgical blood loss would provide multiple benefits: (1) Improved delivery of intraoperative and postoperative care, (2) a quality assurance tool for measuring blood loss for surgeons as part of performance processes are established by surgical departments for re-certification, (3) point of care testing to evaluate when interventions (i.e. transfusion, pharmacologic intervention) need to be initiated, and (4) proper documentation of accurate measure of intraoperative 
blood loss to satisfy quality measures for hospital reporting and accreditation.

A novel tablet computer application that includes Feature Extraction Technology (FET), and utilizes computer vision algorithms to assess hemoglobin concentration contained in surgical sponges through photographic analysis, may satisfy the measures stated above.

Our study hypothesis is that this novel tablet application using FET would be as accurate in measuring hemoglobin loss, as more laborious subjective validated hemoglobin measurement tools with respect to surgical sponges.

\section{MATERIALS AND METHODS}

This study was approved by our Institutional Review Board. Data was gathered in a consecutive series of 50 orthopaedic joint arthroplasty cases. Enrollment was initiated in June 2013 and continued through December 2013 at our clinical site. Preoperative hemoglobin and hematocrit levels as measured by standardized laboratory procedure for each patient were recorded.

Gauss Surgical Inc. (Los Altos, CA) has developed a novel tablet computer application technology, Feature Extraction Technology (FET), that utilizes computer vision algorithms to assess hemoglobin concentration contained in used surgical sponges through photographic analysis. FET software first pre-processed the captured image to both isolates on the sponge surface and the blood-containing regions of interest within the sponge. Given that not all operating rooms contained the same ambient lighting conditions or backgrounds, the program then normalized all images so as to numerically minimize the effects of ambient light. From the pre-processed image, the software extracts a set of geometric and pixel-level features and utilizes a proprietary mathematical model that maps clusters of these features to known hemoglobin $(\mathrm{Hb})$ mass values. These algorithms specifically account for variations in the saline volume absorbed on a sponge, which acts as a diluent and can modify the appearance of a sponge's color.

During the procedures the surgical sponges were removed from the surgical field and photographed "live" (intra-operatively) using a portable tablet, equipped with the Triton system (Gauss Surgical Inc., Los Altos, CA) featuring FET technology, by members of the surgical team resulting in a measurement of hemoglobin mass contained within the sponges (Fig. 1). To operate the Triton System, the Triton application is installed onto the iPad 2 (Apple Inc., Cupertino, CA), a touch-screen operated mobile device with a front-facing camera which is mounted onto IV pole in the operating room. The Triton application captures scans of surgical sponges via the iPad's built-in camera. The capture of each image can be actuated by several means, including a Bluetooth-connected foot-pedal or other methods. Scans of each sponge collected during the surgical case are available for review and editing as needed in real-time. These images are stored on both an onboard and a remote server for processing after the surgical case is completed. FET employs computer vision and machine-learning algorithms are used to automatically filter out the effects of non-sanguineous components absorbed in each sponge or mixed into each canister and computes $\mathrm{Hb}$ mass from the image in real time.
The $\mathrm{Hb}$ mass on each sponge was measured and summed for each case.

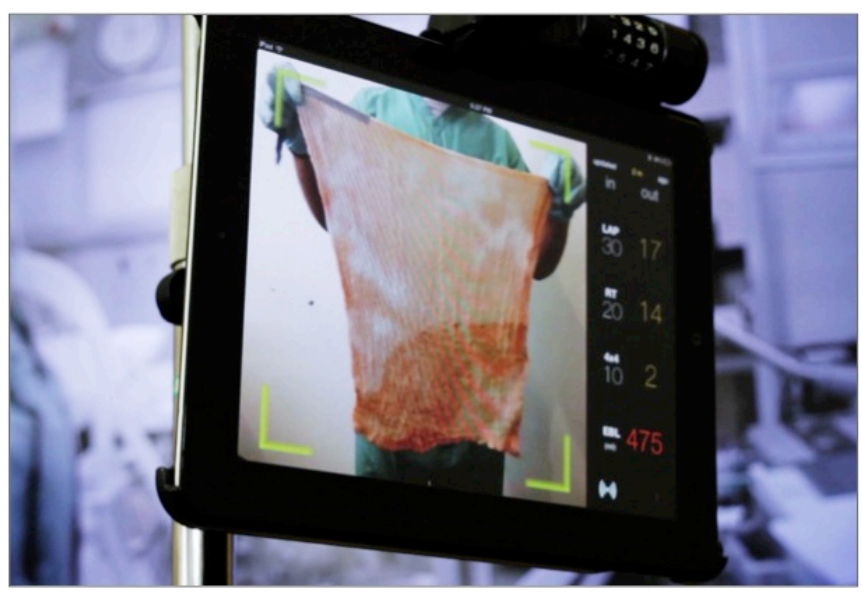

Fig. (1). Image of the Triton System in use during an operation. The nurse holding the examined laparotomy sponge is seen in the screen while the image is captured. The application displays the laparotomy sponge count as well as the individual sponge $\mathrm{Hb}$ amount and the cumulative $\mathrm{Hb}$ loss over the entire operation.

Measures of surgical sponge estimated blood loss (EBL) from the Triton system were compared with the estimates of blood loss via the gravimetric method and photometric analysis which were processed as the following. After the images were scanned by the application, operative sponges were transported to a medical waste room for further assessment. Sponges were rinsed in heparinized solution and dried, with the effluent collecting in a separate container. The container was weighed (recorded in grams) and the hemoglobin concentration (recorded in $\mathrm{g} / \mathrm{ml}$ ) was measured with HemoCue Plasma/Low $\mathrm{Hb}$ photometric system (HemoCue, Brea, CA). Using conversion mathematics, we converted the mass of effluent into milliliters, assuming an average fluid density of $1.0 \mathrm{~g} / \mathrm{ml}$. The recovered mass of hemoglobin contained within the effluent was then calculated by multiplying the concentration of hemoglobin by the total volume of effluent collected. The recovered $\mathrm{Hb}$ mass was scaled by a factor of $85 \%$ to reflect the residual $\mathrm{Hb}$ on sponges, based on previously reported recovery rates of $82 \%$ to $93 \%$ in studies that employed similar methods to recover blood from cotton products [13-16]. This process was completed for each patient.

Since photometric analysis (also referred to as the assay method) is considered the current most accurate method to determine $\mathrm{Hb}$ concentration $[11,12]$ it was used as the reference method to which both the Triton method and Gravimetric method were compared to. To compute estimates of blood loss for the Triton system and the assay method, values of Triton $\mathrm{Hb}$ mass and Assay Hb Mass were divided by the recorded laboratory-derived subject $\mathrm{Hb}$, resulting in values of Triton EBL and Assay EBL. One case was excluded from the analysis of gravimetric method because sponge weights were not recorded. Triton EBL and Gravimetric EBL were then compared to Assay EBL for both correlation and concordance (Bland-Altman).

Software application calculations were then compared to the photometric analysis to assess accuracy and 
reproducibility. No patient contact was necessary for data acquisition and analysis.

\section{Statistical Analysis}

We employed a power analysis to estimate the sample size that would detect a difference in $\mathrm{Hb}$ mass of $30 \mathrm{~g}$ per case, which is approximately half of the hemoglobin content of a unit of allogeneic whole blood. Based on previous studies [17], it was estimated that 47 patients would be necessary in each group (device and reference) to determine a difference of $\pm 30 \mathrm{~g}$ per patient between the Triton $\mathrm{HbL}$ and the assay $\mathrm{Hb}$ mass with $90 \%$ power and a significance level (alpha) of 0.05 . Therefore, this study was planned for 50 patients, each with paired measures of Triton $\mathrm{HbL}$ and the assay $\mathrm{Hb}$ mass (reference standard). To assess the accuracy of the Triton System in these procedures, a concordance analysis was performed using the Bland-Altman framework for agreement between two measurements [18]. Association between the two measurements was studied using linear regression. Blood loss measurements resulting from the $\mathrm{HbL}$ measurements of the Triton system (Triton EBL) and the gravimetric method (Gravimetric EBL) were also compared with a two-sided paired t-test at a significance level of 0.05 .

\section{RESULTS}

\section{Population Characteristics}

A total of 50 cases from a consecutive series of joint arthroplasty cases contributed 857 surgical sponges (18 in $\mathrm{x}$ 18 in, ClearCount Medical, Pittsburgh, USA) for image analysis. The mean (+/- SD) preoperative $\mathrm{Hb}$ was $12.7(+/-$ $1.5) \mathrm{g} / \mathrm{dl}$ and ranged from $8.9 \mathrm{~g} / \mathrm{dl}$ to $15.2 \mathrm{~g} / \mathrm{dl}$. The mean $(+/-\mathrm{SD})$ sponge count per case was $16.8(+/-10)$ and ranged from 3 to 57 sponges per case.

\section{Measurement of $\mathrm{Hb}$ Loss}

A significant positive linear correlation $(r=0.92$ [95\%CI 0.86 to 0.95$]$ ) was noted between the Triton System's measures of sponge $\mathrm{Hb}$ loss per case and the measure of $\mathrm{Hb}$ mass loss obtained from sponges by rinsing and photometric assay of the effluent (reference method). A plot of the correlation between the measures of the Triton System and the reference standard is provided in Fig. (2). The BlandAltman analysis resulted in a bias of $6.4 \mathrm{~g}$ [95\% CI $4.7 \mathrm{~g}$ to $8.2 \mathrm{~g}] \mathrm{Hb}$ per patient. The Bland-Altman limits of agreement (Bias +/- $1.96 \times \mathrm{SD}$ ) indicated that $95 \%$ of all measurements fell within $-5.6(95 \% \mathrm{CI}-7.3$ to -3.9$)$ and 18.5 (95\%CI 16.8 to 20.2) grams of $\mathrm{Hb}$ of photometric analysis, per case (Fig. 3). A quantitative summary of the key accuracy parameters of the Triton System is depicted in Table $\mathbf{1}$.

\section{Measurement of Total Blood Loss}

A strong positive linear correlation between Triton EBL and Photometric EBL per case was observed $(r=0.91$ [95\%CI 0.85 to 0.95$]$ ) (Fig. 4A). Similarly, a moderate positive linear correlation was present between Gravimetric EBL and Photometric EBL per case $(r=0.66$ [95\% CI 0.47 to 0.79]) (Fig. 4B). A Bland-Altman analysis of the Triton

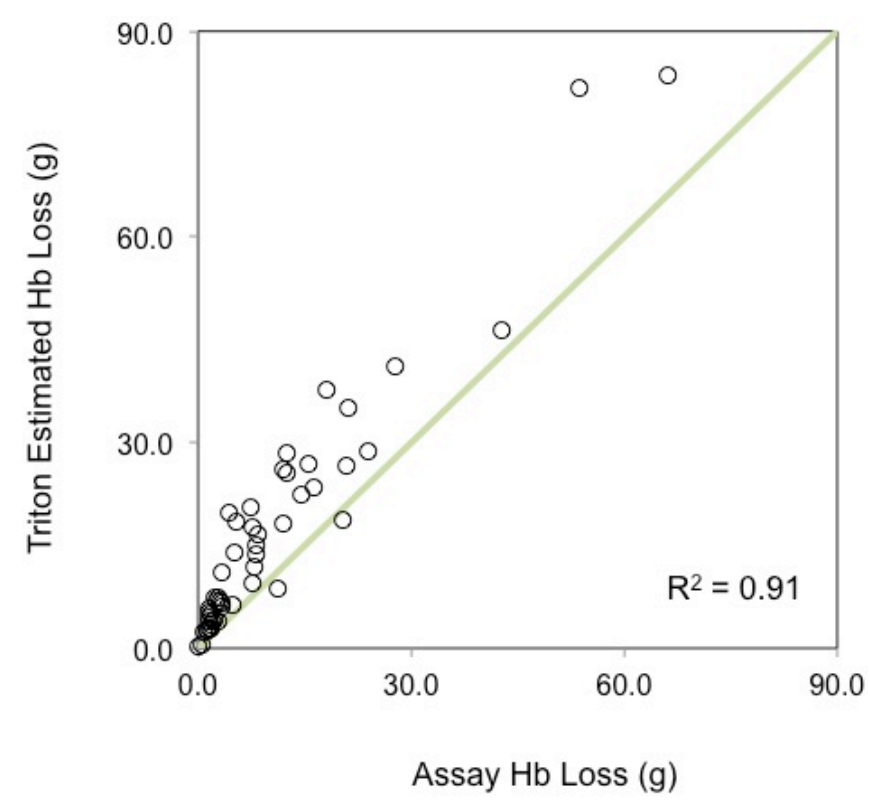

Fig. (2). A plot of the association between the Estimated $\mathrm{Hb}$ loss by the Triton System and the assay $\mathrm{Hb}$ loss using the reference method.

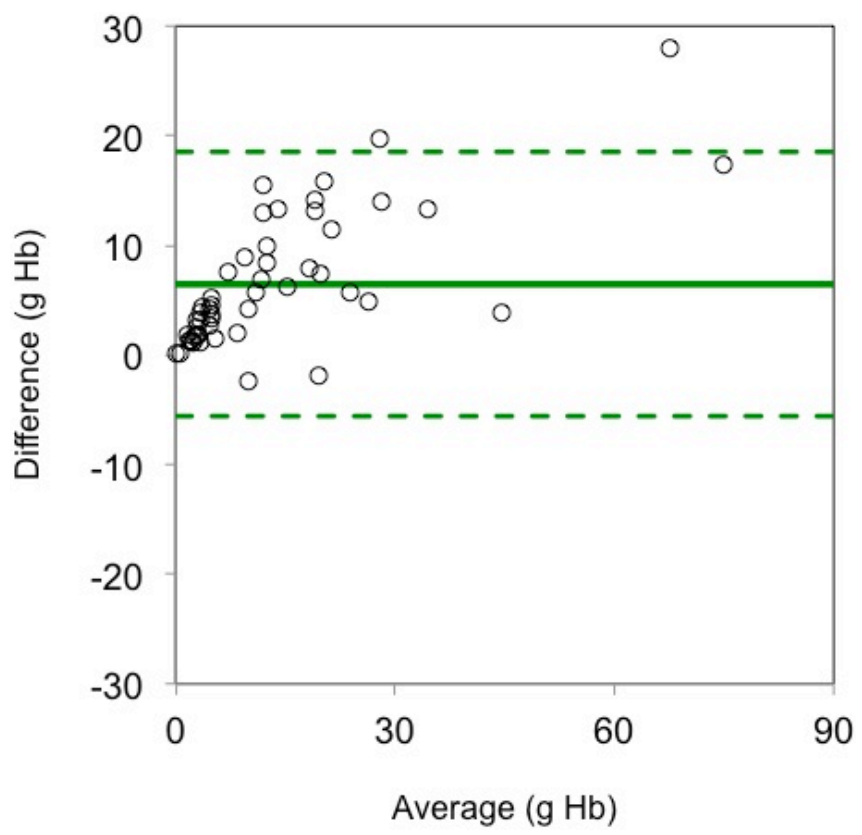

Fig. (3). A Bland-Altman plot of the differences between the tablet application's measures of $\mathrm{Hb}$ loss and the reference measures versus the average of the two measures.

EBL relative to Photometric EBL resulted in a bias of $53 \mathrm{ml}$ $(95 \%$ CI 38 to $68 \mathrm{ml})$ per case. The lower limit of agreement was $-53 \mathrm{ml}(95 \% \mathrm{CI}-68$ to $-37 \mathrm{ml})$ and the upper limit of agreement was $158 \mathrm{ml}(95 \% \mathrm{CI} 143$ to $173 \mathrm{ml})$, for the $95 \%$ concordance level between the Triton system and photometric analysis. Similarly a Bland-Altman analysis of the Gravimetric EBL relative to the photometric EBL resulted in a bias of $283 \mathrm{ml}(95 \% \mathrm{CI} 234$ to $331 \mathrm{ml})$ per case. The lower limit of agreement was $-51 \mathrm{ml}(95 \% \mathrm{CI}-100$ to $3 \mathrm{ml})$ and the upper limit of agreement was $616 \mathrm{ml}(95 \% \mathrm{CI}$ 568 to $665 \mathrm{ml}$ ), for the $95 \%$ concordance level between the Gravimetric method and photometric analysis. A paired t-test 
Table 1. Performance summary of the association and agreement between the Triton System and the reference method, compared with the performance of the Gravimetric method.

\begin{tabular}{|c|c|c|c|}
\hline \multirow{2}{*}{ Parameter } & \multicolumn{2}{|c|}{ Triton System } & \multirow{2}{*}{$\begin{array}{c}\text { Gravimetric Method } \\
\text { Blood Loss (ml) }\end{array}$} \\
\hline & Hb Loss (g) & Blood Loss (ml) & \\
\hline Mean sponge count (SD) & $17(10)$ & $17(10)$ & $17(10)$ \\
\hline Range & 0.1 to 83.6 & 1 to 716 & 22 to 1312 \\
\hline Correlation, r $(95 \%$ CI) & $0.92(0.86$ to 0.95$)$ & $0.91(0.85$ to 0.95$)$ & $0.66(0.47$ to 0.79$)$ \\
\hline RMSE & 8.9 & 75 & 329 \\
\hline SD (difference) & 6.1 & 54 & 170 \\
\hline Bias $(95 \% \mathrm{CI})$ & $6.4(4.7$ to 8.2$)$ & $53(38$ to 68$)$ & $283(234$ to 331$)$ \\
\hline Lower limit of agreement $(95 \% \mathrm{CI})$ & $-5.6(-7.3$ to -3.9$)$ & $-53(-68$ to -37$)$ & $-51(-100$ to -3$)$ \\
\hline Upper limit of agreement $(95 \% \mathrm{CI})$ & 18.5 (16.8 to 20.2$)$ & $158(143$ to 173$)$ & $616(568$ to 665$)$ \\
\hline
\end{tabular}

revealed that mean Gravimetric EBL exceeded mean Triton EBL by $228 \mathrm{ml}$ per case $(363 \mathrm{ml} v s 135 \mathrm{ml}, \mathrm{p}<0.0001)$.

\section{DISCUSSION}

Our results showed a significant positive correlation between Triton tablet system and the referenced photometric analysis with respect to intra-operative hemoglobin and blood loss at 0.92 and 0.91 , correlations respectively. Although there was a bias of $6.4 \mathrm{~g}$ of hemoglobin between the Triton system and photometric analysis, as evidenced through a Bland-Altman plot, this value approximates to one-tenth of a standard unit of whole blood $(450 \mathrm{ml})$ with a hemoglobin concentration of $12.7 \mathrm{~g} / \mathrm{dl}$, which was the average $\mathrm{Hb}$ value in our study. This discrepancy is not clinically significant because $45 \mathrm{ml}$ of blood represents $0.9 \%$ of the circulating volume of the standard $70 \mathrm{~kg}$ male with five liters of intravascular volume.
When the tablet software and gravimetric method were compared to the photometric analysis for direct measurement of intraoperative blood loss, the tablet proved superior with 0.91 versus 0.66 correlations, respectively. As discussed above, confounding variables like serous bodily fluids, saline irrigation and solid tissue that can alter the "wet weight" of surgical sponges are the likely cause for the decreased correlation of the gravimetric method. Although the gravimetric method has proven to be less labored intensive than photometric analysis, our results show that it was significantly less accurate when compared to the tablet software, with biases of $283 \mathrm{ml}$ versus $53 \mathrm{ml}$, respectively. This six-fold increase in bias makes the gravimetric method unreliable in the presence of a more accurate technique in the measurement of intraoperative blood loss.

Despite these methods for measuring hemoglobin and total blood loss, it is still commonplace to rely on visual estimation due to convenience and ease. This method has proven to be inaccurate and often underestimates the amount
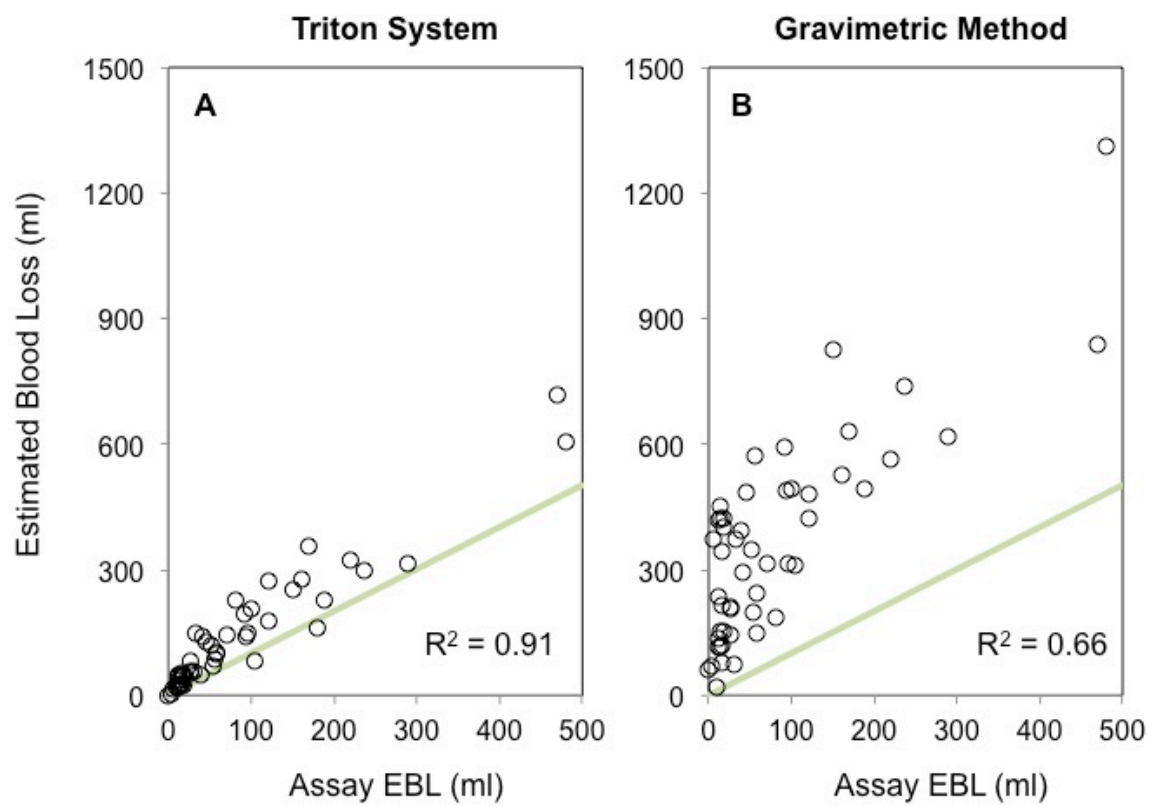

Fig. (4). Accuracy comparison of Estimated Blood Loss (EBL, ml) measured via the Triton System and the Gravimetric method, against the reference method. a) Triton EBL and Assay EBL per case, b) Gravimetric EBL and Assay EBL per case. 
of blood loss in multiple studies, yet remains the standard in many operating rooms $[6,7,9,19]$. It has been shown that simulations and didactic training can mildly improve estimation methods, however these benefits are short-lived and experience of the physician does not improve estimation values overall $[3,20]$.

One limitation of this study was the lack of a true "gold standard" in measuring blood loss, since the rinsing of sponges to assay $\mathrm{Hb}$ content (photometric analysis) does not guarantee $100 \%$ recovery of $\mathrm{Hb}$. However, we attempted to control for the inherent bias of this method by (1) standardizing the rinsing protocol (cycle times, diluent volumes, centrifuge dimensions), and (2) adjusting the assayed values by a factor of $15 \%$ based on prior studies [1317]. Such limitations notwithstanding, this novel tablet technology provides surgeons, anesthesiologists, and other operative personnel with a real-time measurement of blood loss so that intraoperative actions can be taken based on objective real time measurements rather than subjective analysis that can be confounded by numerous variables like intravenous fluid flow rate, amount and rate of flow of blood loss, intraoperative pharmaceuticals, and other factors that can directly impact the circulatory drive of the patient.

We, therefore, conclude that our results support the accuracy of this novel tablet software as a real-time measurement to accurately quantify intraoperative blood loss that has been gathered on surgical sponges. Further studies are currently underway using the Triton system for accurate assessment of hemoglobin content within suction canisters which will allow calculation of to total intraoperative blood loss.

\section{CONFLICT OF INTEREST}

BS, SW, PA, RS- have no disclosures and conflicts, SSis an employee of Gauss Surgical.

\section{ACKNOWLEDGEMENTS}

Declared none.

\section{REFERENCES}

Bonica JJ LC. Measurement of blood loss during surgical operations. Am J Surg 1951; 81(5): 496-502.
[2] Meiser A, Casagranda O, Skipka G, Laubenthal H. Quantification of blood loss. How precise is visual estimation and what does its accuracy depend on? Der Anaesthesist 2001; 50(1):13-20.

[3] Toledo P, McCarthy RJ, Hewlett BJ, Fitzgerald PC, Wong CA. The accuracy of blood loss estimation after simulated vaginal delivery. Anesth Analgesia 2007; 105(6):1736-40.

[4] Ashburn JC, Harrison T, Ham JJ, Strote J. Emergency physician estimation of blood loss. West J Emerg Med 2012; 13(4): 376-9.

[5] Brecher ME MT, Goodnough LT. A standardized method for calculating blood loss. Transfusion 1997; 37: 1070-4.

[6] McCullough TC, Roth JV, Ginsberg PC, Harkaway RC: Estimated blood loss underestimates calculated blood loss during radical retropubic prostatectomy. Urol Int 2004; 72(1): 13-6.

[7] Seruya M, Oh AK, Boyajian MJ, Myseros JS, Yaun AL, Keating RF: Unreliability of intraoperative estimated blood loss in extended sagittal synostectomies. J Neurosurg Pediatr 2011; 8(5): 443-9.

[8] Kolb KS, Day T, McCall WG: Accuracy of blood loss determination by health care professionals. CRNA 1999, 10(4): 170-173.

[9] Budny PG, Regan PJ, Roberts AH. The estimation of blood loss during burns surgery. Burns 1993, 19(2): 134-7.

[10] Johar RS, Smith RP. Assessing gravimetric estimation of intraoperative blood loss. J Gynecol Surg 1993; 9(3): 151-4.

[11] Guinn NR, Broomer BW, White W, Richardson W, Hill SE. Comparison of visually estimated blood loss with direct hemoglobin measurement in multilevel spine surgery. Transfusion 2013, 53(11): 2790-4.

[12] Keenan WN, Griffiths H, Clegg J. Evaluating blood loss in children's orthopaedic surgery: a simplified method of photometric analysis of eluted swabs. J Pediatr Orthop 1998; 18(4):488-91.

[13] Hill SE, Broomer B, Stover J, White W, Richardson W. Bipolar tissue sealant device decreases hemoglobin loss in multilevel spine surgery. Transfusion 2012;52(12): 2594-9.

[14] van Eijkeren MA, Scholten PC, Christiaens GC, Alsbach GP, Haspels AA: The alkaline hematin method for measuring menstrual blood loss--a modification and its clinical use in menorrhagia. Eur J Obstet Gynecol Reprod Biol 1986; 22(5-6): 345-51.

[15] Newton J, Barnard G, Collins W. A rapid method for measuring menstrual blood loss using automatic extraction. Contraception 1977; 16(3): 269-82.

[16] Magnay JL, Nevatte TM, Dhingra V, O'Brien S. Menstrual blood loss measurement: validation of the alkaline hematin technique for feminine hygiene products containing superabsorbent polymers. Fertil Steril 2010; 94(7): 2742-6.

[17] Hill SE, Stover J, White W. Accuracy of estimated blood loss in spine suregery. In: American Society of Anesthesiologists Annual Meeting. Chicago, IL 2011.

[18] Bland JM, Altman DG. Statistical methods for assessing agreement between two methods of clinical measurement. Lancet 1986; 1(8476): 307-10.

[19] Schorn MN. Measurement of blood loss: review of the literature. J Midwife Women Health 2010; 55(1): 20-7.

[20] Dild GA 3rd, Paine AR, George NC, Velasco C. Estimating blood loss: can teaching significantly improve visual estimation? Obstet Gynecol 2004; 104(3): 601-6. 